[0212-7199 (2005) 22: 5; pp 227-230] ANALES DE MEDICINA INTERNA Copyright (C) 2005 ARAN EDICIONES, S.L

AN. MED. INTERNA (Madrid) Vol. 22, N. ${ }^{\circ}$ 5, pp. 227-230, 2005

\section{Enterolitiasis múltiple, coexistiendo con litiasis biliar y vesical, asociada a adenocarcinoma de colon}

\author{
J. A. ORTS ${ }^{1}$, L. MORELL ${ }^{1,2}$, J. CAMPS ${ }^{3}$, M. L. TRABA ${ }^{4}$, A. BELENGUER ${ }^{5}$, \\ A. GUERRERO ${ }^{1}$
}

'Área de Diagnóstico Biológico. ${ }^{2}$ Servicio de Anatomía Patológica. ${ }^{3}$ Servicio de Radiología. ${ }^{5}$ Servicio de Medicina Interna. Hospital de la Ribera. Alzira, Valencia. ${ }^{4}$ Laboratorio de Bioquímica. Sección Fisopatología Ósea. Fundación Jiménez Díaz. Madrid

\section{RESUMEN}

La enterolitiasis, cálculos formados primariamente en el intestino, es una rara enfermedad asociada, generalmente, con estasis intestinal. Suele cursar sin síntomas en la mayoría de los casos, pero puede presentar oclusión intestinal y debe ser tenida en cuenta como posible causa de la misma. Presentamos, un caso de enterolitiasis múltiple, patología muy infrecuente, que coexiste con litiasis biliar y vesical en un paciente con adenocarcinoma de colon. El diagnóstico fue realizado por imágenes de Rx y TC. El análisis de los cálculos: químico, por espectroscopia infrarroja, microscopía estereoscópica y espectrometría de emisión atómica, mostró una composición basada en materia orgánica y whilokita (ortofosfato de calcio y magnesio). No se encontraron factores de riesgo para la litogénesis, exceptuando la estasis intestinal ocasionada por la estenosis provocada por el adenocarcinoma. Se sugiere que los factores genéticos podrían ser los principales contribuyentes a la hiperlitogénesis observada en este caso. Profundizamos en el proceso fisiopatológico y revisamos la literatura al respecto.

PALABRAS CLAVE: Enterolitiasis. Cálculo intestinal. Obstrucción intestinal.
MULTIPLE ENTEROLITHIASIS, COEXISTING WITH BLADDER AND GALL BLADDER LITHIASIS, ASSOCIATED WITH COLON ADENOCARCINOMA

\section{ABSTRACT}

Enteroliths are calculi primarily formed in the intestine. Enterolithiasis is a rare condition frequently associated with intestinal stasis. Usually it causes no symptoms in most cases, but it can be an important diagnostic clue in patients presenting intestinal occlusive symptoms. We report a case of multiple enterolithiasis, very infrequent pathology, coexisting with bladder and gall bladder lithiasis in a patient with colon adenocarcinoma. Diagnosis was made by X-rays and CT images. Calculi were analysed by several methods: chemical, infrared spectroscopy stereoscopic microscopy and atomic emission spectroscopy; they showed that caluli are made up of organic material and whilokita (calcium and magnesium ortophosphate). No risk factors for lithogenesis were found in this patient excluding the intestinal stasis caused by intestinal narrowing as a result of adenocarcinoma. Genetic factors are suggested as main contributors to hyperlithogenesis observed in this patient. The physiopathological conditions were studied in depth and literature about this subject reviewed.

KEY WORDS: Enterolithiasis. Intestinal calculi. Intestinal obstruction.

Orts JA, Morell L, Camps J, Traba ML, Belenguer A, Guerrero A. Enterolitiasis múltiple, coexistiendo con litiasis biliar y vesical, asociada a adenocarcinoma de colon. An Med Interna (Madrid) 2005; 22: 227-230.

\section{INTRODUCCIÓN}

La enterolitiasis, o presencia de cálculos en el intestino, es una entidad clínica observada muy raramente en seres humanos aunque, por el contrario, común en equinos en los que se asocia a factores de riesgo múltiples: dietéticos, ambientales y genéticos. Todavía más infrecuente es la presencia de enterolitiasis múltiple, y si a ello sumamos la de litiasis biliar y vesical, nos encontramos con un caso excepcional como el que presentamos, del que no hemos hallado precedente en la literatura.

Normalmente, los enterolitos son observados como hallazgos radiológicos y se asocian a estenosis intestinal. La enterolitiasis debe ser considerada en el diagnóstico diferencial en pacientes con síntomas abdominales.
Describimos un caso de enterolitasis múltiple asociado a adenocarcinoma de colon en un anciano, revisamos la literatura al respecto y profundizamos en la fisiopatología del proceso.

\section{CASO APORTADO}

Un varón de 91 años que ingresó a través del Servicio de Urgencias, refieriendo dolor y molestias abdominales, presentaba vómitos. A la exploración, el abdomen se mostró distendido, blando, timpánico, depresible, con asas dilatadas visibles a la inspección, sin puntos dolorosos. No existían antecedentes médicos de interés. Presentaba una cicatriz quirúrgica por herida de guerra. No refería reacciones adversas a medicamentos, ni hábitos tóxicos. Se observó gonartrosis bilateral e hipertrofia benigna de prostata grado III. Presentó un

Trabajo aceptado: 10 de diciembre de 2004

Correspondencia: J. A. Orts. Área de Diagnóstico Biológico. Hospital de la Ribera. Ctra. Alzira-Corbera, km. 1. 46600 Alzira (Valencia) e-mail: jaorts@ @ospital-ribera.com 
ingreso hace 10 años por obstrucción intestinal, pero no aportó informes. Había sido intervenido de cataratas y hernia inguinal. Como medicación sólo tomaba laxantes (plantago ovata, Plantaben ${ }^{\circledR}$ ) y parafina líquida, Hodernal ${ }^{\circledR}$ ). Manifestó incontinencia urinaria habitual y fecal ocasional.

La Rx simple de abdomen mostraba asas de intestino delgado muy dilatadas en hemiabdomen izquierdo y multiples imágenes de densidad calcio con centro radiolúcido proyectadas sobre las asas de intestino delgado y el ciego, asimismo dilatado. También se observaron niveles hidroaéreos en intestino delgado y ciego compatibles con patrón obstructivo (Fig. 1).

Las imágenes de TAC abdominal revelaban enterolitos en ciego, asas de ileon y yeyuno. También se apreciaron quistes hidatídicos hepáticos (uno de $10 \times 9 \mathrm{~cm}$ en segmentos VII-V, no calcificado y otro de $46 \mathrm{~mm}$ en segmento I, parcialmente calcificado). Igualmente, se observaba litiasis en vesicula biliar y litiasis multiple en vejiga urinaria.

Nos encontramos, sorprendentemente, ante un paciente hiperlitogénico generador de cálculos en el tracto intestinal, biliar y urinario. Los enterolitos se situaban en el ciego y a lo largo de toda la luz del intestino delgado, acumulados preferentemente en el íleon terminal, el cual mostraba una dilatación preestenótica de 13 x $10 \mathrm{~mm}$. (Figs. 2 a, b, c, d).

Se intervino quirúrgicamente, encontrándose una neoplasia estenosante en el ángulo hepático de colon, sin afectar a la serosa, con presencia de adenopatías en todo el meso hasta raíz de vasos. Se procedió a hemicolectomía derecha con ileotransversostomía.

El estudio anatomopatológico macroscópico mostró una tumoración estenosante y ulcerada en colon derecho, de 4x1,5x1 cm y una gran dilatación cecal en una extensión de $10 \mathrm{~cm}$, con pérdida de plie-

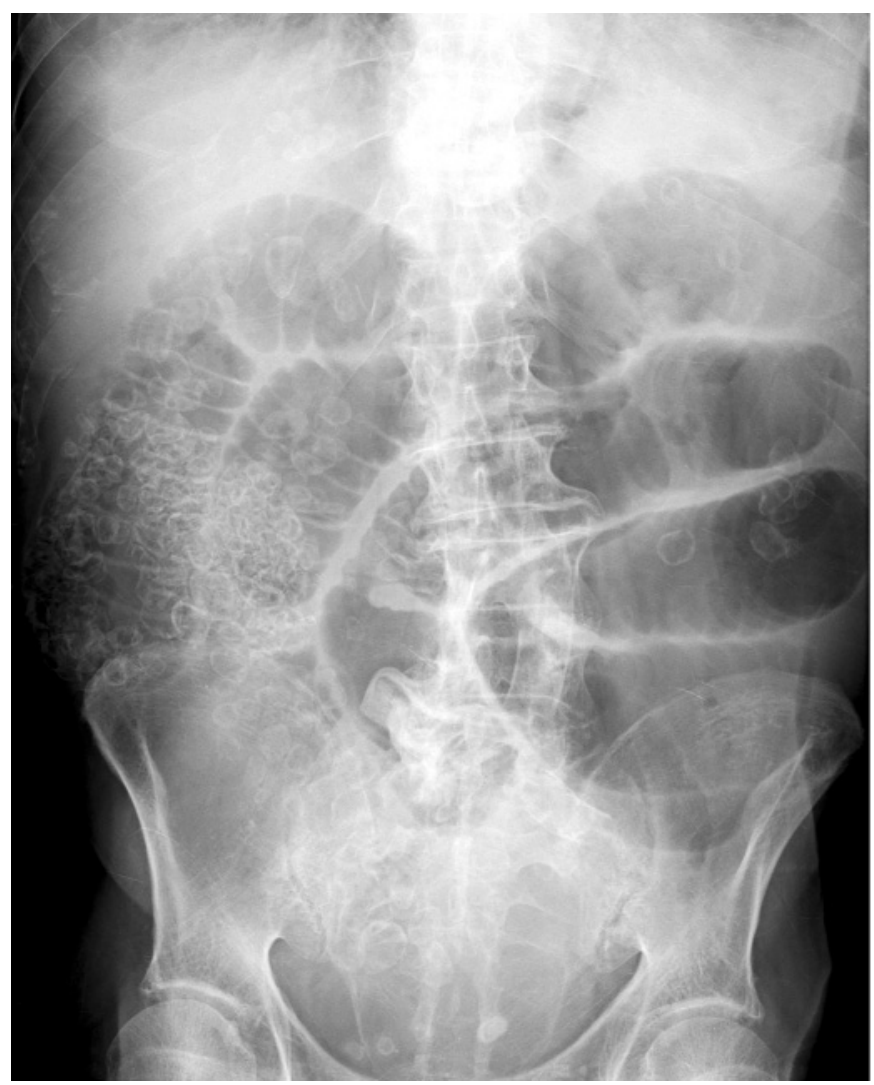

Fig. 1. Diagnóstico por imagen de la enterolitiasis múltiple. Rx simple de abdomen donde se aprecia dilatacion de asas de intestino delgado y múltiples imágenes de densidad calcio con centro radiolúcido proyectadas sobre las asas de intestino delgado y el ciego, asimismo dilatado. gues intestinales y seudotabicación, mucosa congestiva y una gran cantidad de coprolitos (259) afacetados de tamaño comprendido entre 2 y $20 \mathrm{~mm}$, de formas diversas aunque preferentemente redondeados, que en conjunto ocupan un volumen aproximado de $250 \mathrm{cc}$ (Figs. 3 y 4). Histológicamente correspondió a adenocarcinoma (pT3G1) sin otras lesiones microscópicas relevantes.

El análisis químico de los enterolitos mostró que estaban constituidos por calcio, fosfato y magnesio; por el contrario carecían de colesterol y bilirrubina. Por análisis de espectrometría infrarroja se identificaron como carbonato apatita de tipo whitlokita (ortofosfato de calcio y magnesio) y materia orgánica. Por análisis polarográfico diferencial se confirmó la whitlokita sobre un substrato de materia orgánica. El análisis por espectrometría de emisión atómica reafirmó la composición mayoritaria de calcio, fosforo y magnesio.

Se realizó una pormenorizada historia clínica incidiendo en la anamnesis de cualquier factor de riesgo litógeno. Así, para la litiasis biliar se valoró: obesidad, historia familiar, diabetes mellitus, hipertensión, dislipemia, enfermedad cardiaca coronaria, enfermedad cerebrovascular, hábito tabáquico y alcohólico. Para la litiasis renal: dieta, ingesta hídrica, hiperparatiroidismo, hipercalciuria, hiperoxaluria, hiperuricuria, hipocitraturia. Por último, se interrogó sobre el uso de fármacos (especialmente antiácidos). Sin embargo, las datos obtenidos no fueron significativos, ni pudieron aportar justificación alguna a la hiperlitogénesis del paciente.

\section{DISCUSIÓN}

Los enterolitos, ya conocidos desde antiguo aunque poco estudiados, fueron definidos por Atwell y Pollock en 1960 (1), como cuerpos extraños formados primariamente en el intestino. Suelen estar constituidos por concreciones minerales (fundamentalmente sales de calcio y magnesio) y materia orgánica. El primer registro radiológico data de 1917. Un tercio de los enterolitos son radiopacos en la periferia donde se deposita la mayoría de los minerales y radiolúcidos en el núcleo. La visibilidad de los mismos depende de su contenido en calcio, se considera que el medio alcalino del íleo distal favorece su génesis. Los enterolitos radiolúcidos suelen estar formados por ácidos biliares (ácido cólico) y se forman, con frecuencia, en el duodeno o yeyuno en medio ácido $(2,3)$.

Los enterolitos son usualmente únicos, pero a veces se encuentran en número de 2 a 9 y muy infrecuentemente en grandes cantidades. Se han clasificado como primarios, formados propiamente en el intestino, o secundarios cuando se generan fuera del mismo, como los cálculos biliares. También se consideran como verdaderos o falsos (si provienen de la ingesta de material extraño indigerible (bezoar), como pelo, fibra, o barniz) en cuyo caso también se pueden encontrar en el estómago. También se ha descrito que pueden nuclearse sobre semillas de frutos.

Los casos publicados de enterolitiasis en humanos han sido asociados a estasis intestinal (principal factor predisponente para su formación) por estenosis, ya disgenésica (19 casos de disgenesia ileal) (3) o por diversas causas: apéndice, diverticulosis, incluyendo divertículo de Meckel $(4,5)$ o no de Meckel (en duodeno, yeyuno, íleo y quistes duplicados ileales); asociada a enfermedad de Crohn (23 casos publicados) (2), a tuberculosis intestinal, a tumor carcinoide, a enteritis por radiación, estrechamiento funcional intestinal, y post-traumática o post-quirúrgica. La gran mayoría de los pacientes presentaban los enterolitos en el intestino delgado. En un estudio de 84 casos con diverticulo de Meckel, se observaron cálculos en 8 de los pacientes estudiados (10\%) (6); aunque en 

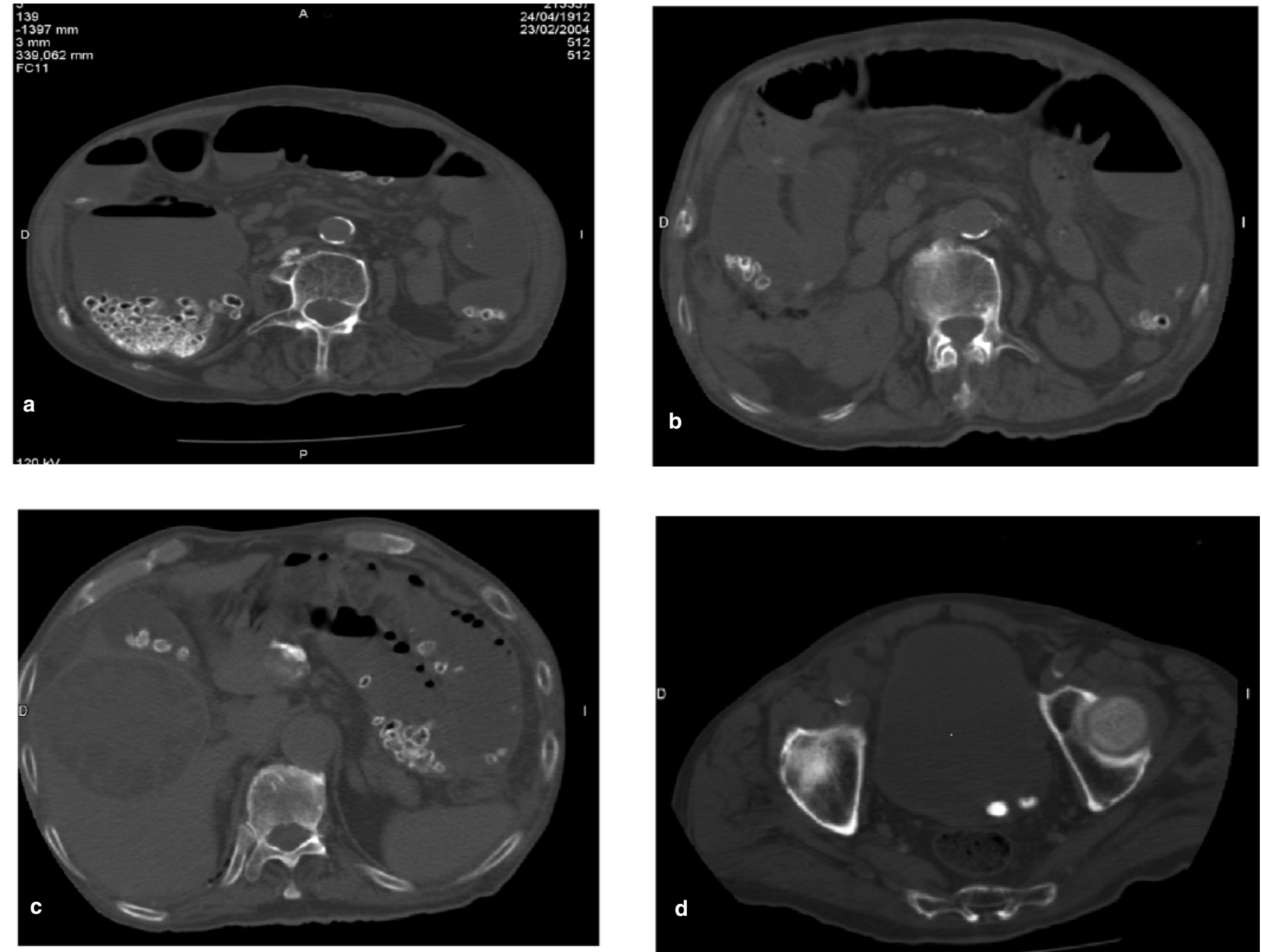

Fig. 2. Imágenes de TC. a: se aprecian los enterolitos en ciego y asas de yeyuno. b: en asas de íleon y yeyuno. c: en asas de yeyuno, así como litiasis en vesicula biliar e incidentalmente, un quiste hidatídico hepático. d: pueden observarse litiasis vesicales.

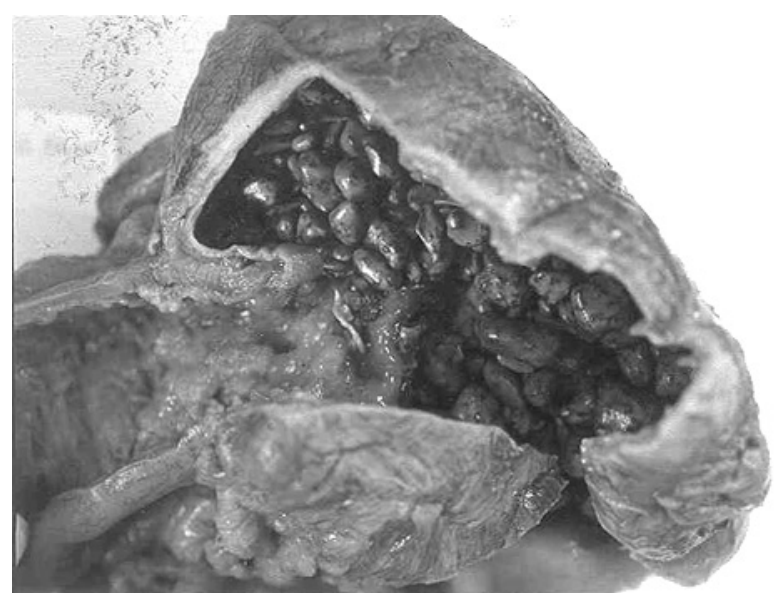

Fig. 3. Fragmento de colon resecado conteniendo enterolitos.

otro sobre 776 casos quirúrgicos, sólo encuentran dos de enterolitiasis (7). Se ha descrito en un paciente afecto de esprue no tropical. Sin embargo, sólo se ha publicado un caso de enterolitasis masiva, asociada a disgenesia ileal (3) .

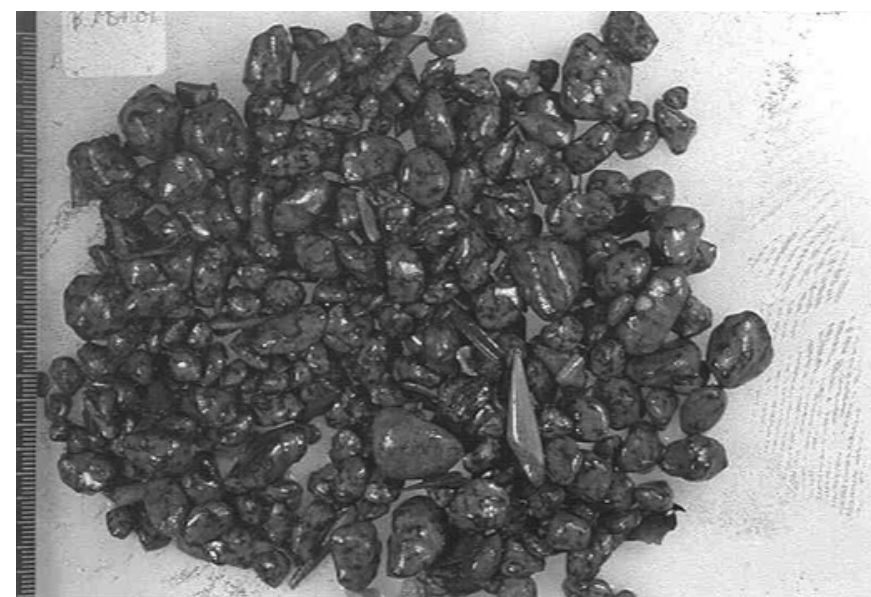

Fig. 4. Enterolitos.

Aunque los enterolitos en la mayoría de los casos no manifiestan sintomatología, suelen considerarse como consecuencia de una enfermedad crónica o subaguda intestinal, más que una causa de obstrucción, sin embargo pueden ocasionar estrechamiento intestinal significativo y, en algún caso, inclu- 
so obstrucción $(8,9)$. En los casos asociados a enfermedad de Crohn de larga duración se ha sugerido que pueden ser indicativos de un adenocarcinoma subyacente.

Los enterolitos calcificados, observados por técnicas de imagen, pueden ser extremadamente móviles a causa del desplazamiento del mesenterio o de su migración a lo largo de la luz intestinal. La forma oval o redondeada y laminada, observada mediante Rx, permite diferenciarlos de otras causas de calcificación: nódulos linfáticos mesentéricos, o calcificaciones pancreáticas. El estudio de doble contraste con enema de bario mejora la identificación y localización de los cálculos. Mediante TC, podremos observar aquellos enterolitos pobremente calcificados y radiolúcidos a $\mathrm{Rx}(2,3)$.

Pese a haber realizado un riguroso estudio en el paciente de todos los factores de riesgo litógeno conocidos, ninguno de ellos se ha mostrado suficientemente relevante. Por otra parte, recientemente se ha sugerido la posible participación los mecanismos de intercambio electrolítico en la génesis de enterolitos en caballos, concretamente se ha postulado -en un estudio todavía no publicado- la posible participación de los intercambiadores sodio/hidrógeno y cloruro/bicarbonato, pensándose que una significativa reducción en la expresión de los genes que regulan estos procesos podría favorecer la susceptibilidad de esta patología. Además, los factores genéticos han sido suficientemente establecidos en la calculogénesis renal y biliar (10-12). En el caso presentado, la hiperlitogénesis sin etiología conocida, aunque favorecida por la estenosis carcinomatosa, apoya la hipótesis de un sustrato genético alterado en el paciente que predisponga a una manifiesta susceptibilidad a la calculogénesis.

\section{Bibliografía}

1. Atwell JD, Pollock AV. Intestinal calculi. Br J Surg 1959-1960; 47 : 367-374.

2. Mendes Ribeiro HK, Nolan DJ. Enterolithiasis in Crohn's disease. Abdom Imaging 2000; 25: 526-529.

3. Grassi R, Pinto A, Valente T, Rossi G, Catalano O, Rotondo A, et al. Massive enterolithiasis associated with ileal dysgenesis. Br J Radiol 1997; 70: 207-9.

4. Sáenz de Ormijana J, Aisa P, Añorbe E, Olabarría I. Diverticulitis de Meckel perforada con enterolito: diagnóstico mediante ecografía y TC. Radiología 2001; 43: 109-111.

5. Wouter van Es H, Sybrandy R. Case 19: Enteroliths in a Meckel diverticulum. Radiology 2000; 214: 524-526.

6. Pantongrag-Brown L, Levine MS, Buetow PC, Buck JL, Elsayed AM. Meckel's enteroliths: clinical, radiologic, and pathologic findings. Am J Roentgenol 1996; 167: 1447-50.

7. Kusumoto H, Yoshida M, Takahashi I, Anai H, Maehara Y, Sugimachi
K. Complications and diagnosis of Meckel's diverticulum in 776 patients. Am J Surg 1992; 164: 382-3.

8. Raidu BRS, Patnaik S, Rao CLV. Enteroliths. Disponible en: http: //www.ijri.org/articles/archives/2002-12-1/letters_135.htm.

9. Klingler PJ, Seelig MH, Floch NR, Branton SA, Metzger PP. Smallintestinal enteroliths - unusual cause of small-intestinal obstruction: report of three cases. Dis Colon Rectum 1999; 42: 676-9.

10. Hassel DM, Snyder JR, Aldridge BM, Stott JL. Identification of genes involved in equine enterolith formation. Disponible en: htttp: //www.vetmed.ucdavis.edu/ceh/RR03GEN00-25.html.

11. Langman CB. The molecular basis of kidney stones. Curr Opin Pediatr 2004; 16: 188-93.

12. Nakeeb A, Comuzzie AG, Martin L, Sonnenberg GE, Swartz-Basile D, Kissebah AH, Pitt HA. Gallstones: genetics versus environment. Ann Surg 2002; 235: 842-9. 